\title{
Myocarditis following mRNA-Based COVID-19 Vaccines: Correspondence
}

\author{
Rujittika Mungmunpuntipantip ${ }^{a}$ Viroj Wiwanitkit ${ }^{b}$ \\ aprivate Academic Consultant, Bangkok, Thailand; ${ }^{b}$ Honorary Professor, Dr. D.Y. Patil University, Pune, India
}

\section{Dear Editor,}

We would like to share ideas on the publication "Incidence, clinical presentation and management of myocarditis following mRNA-based Covid-19 vaccines: A brief report [1]." Saeed et al. [1] concluded that "myocarditis following $m R N A$-based Covid-19 vaccines is rare, more frequently affects younger men $<30$ years and ... outweighs the risk of post-vaccination myocarditis. [C1]." The COVID-19 vaccine, we agree, has the potential to induce negative effects. A heart issue may occur in the patient, which may or may not be related to the COVID-19 vaccination. Because there is no information about the patient's immunological status before to immunization, it is impossible to make a firm conclusion. Several possible underlying pathomechanisms including abnormal immune response and viscosity charge are proposed as an underlying cause of a cardiac problem. It is also likely that a vaccination recipient might experience a medical issue at the same time. For example, among COVID-19 vaccine recipients, dengue fever is a possible concurrent medical condition that might cause myocarditis [2]. Finally, due to the mild nature of the heart condition, there may be underreported cases around the world. Therefore, the discussed incidence by Saeed et al. [1] should be lower than the exact incidence. Despite the lack of a precise management recommendation, we believe that patient followup is beneficial.

\section{Statement of Ethics}

Not applicable.

\section{Conflict of Interest Statement}

The authors have no conflicts of interest to report.

\section{Funding Sources}

No funding was received for this letter.

\section{Authors contributions}

R.M. contributed to $50 \%$ of the following: 1a substantial contributions to study conception and design; 1b. substantial contributions to acquisition of data; $1 \mathrm{c}$. substantial contributions to analysis and interpretation of data; 2 . drafting the article or revising it critically for important intellectual content; and 3. final approval of the version of the article to be published. V.W. contributed to $50 \%$ of the following: 1a substantial contributions to study conception and design; $1 \mathrm{~b}$. substantial contributions to acquisition of data; 1c. substantial contributions to analysis and interpretation of data; 2. drafting the article or revising it critically for important intellectual content; and 3. final approval of the version of the article to be published.

\section{References}

1 Saeed S, Käsk L, Rajani R, Larsen TH. Incidence, clinical presentation and management of myocarditis following mRNA-based Covid-19 vaccines: a brief report. Cardiology. 2022 Feb 1. Online ahead of print.

2 Kebayoon A, Wiwanitkit V. Dengue after COVID-19 vaccination: possible and might be missed. Clin Appl Thromb Hemost. 2021 Jan-Dec;27: 10760296211047229 . 